\title{
REACTIVE MILIEU OF HODGKIN LYMPHOMA WITH EMPHASIS ON MAST CELLS AND MACROPHAGES
}

\author{
Nidhish Kumar', Radha Ram Pai', Isra Halim³ \\ ${ }_{1}^{1}$ Postgraduate Student, Department of Pathology, Kasturba Medical College, Mangalore. \\ 2 Professor, Department of Pathology, Kasturba Medical College, Mangalore. \\ 3Postgraduate Student, Department of Microbiology, Kasturba Medical College, Manipal.
}

ABSTRACT

\section{AIM OF STUDY}

To study the clinical importance of reactive microenvironment in Hodgkin Lymphoma (HL) with special reference to macrophages and mast cells.

\section{MATERIALS AND METHODS}

The present prospective and retrospective study was undertaken for a period ranging from January 2011 to June 2015 at the Department of Pathology, Kasturba Medical College, Mangalore. The Haematoxylin and Eosin (H and E) stained slides were reviewed and classified using WHO (2008) classification. Six immuno-histochemical markers were used in the study. CD 68 was for the macrophage count. Giemsa stain was done to highlight the mast cells.

\section{RESULTS AND ANALYSIS}

Thirty cases of HL were studied. Out of the 5 cases of Lymphocyte Depleted (LD) Classical Hodgkin Lymphoma (cHL), all cases showed high macrophage count. Out of 30 cases of HL, only 6 cases showed increased mast cell count.

\section{DISCUSSION}

Mast cells act actively in various types of cancers. They can either have a pro-tumorigenic function or an anti-tumorigenic function depending on the type of cancer. Four (80\%) cases of LD-cHL showed macrophage count between 25-50\% and 1 (20\%) case showed macrophage count $>50 \%$ correlating with the aggressive nature and advanced stage of the disease.

\section{CONCLUSION}

In this study of microenvironment of HL mast cells and macrophages were analysed in each subtype. Though the mast cells were seen in all cases, an increased count of $>10 / 10$ (High power field) HPF was observed only in 6 cases. The macrophage count was highest in LD-cHL and was statistically significant and thus correlated with this aggressive subtype of HL. The mast cell and macrophage count did not correlate with B-symptoms and stage of the disease a conclusion on survival versus the macrophage count and mast cell count was not possible in this study because of shorter follow up. A longer follow up and more number of cases are needed for a significant outcome.

\section{KEYWORDS}

Hodgkin Lymphoma (HL), Immunohistochemistry (IHC), Classical Hodgkin Lymphoma (CHL), Nodular Sclerosis (NS), Mixed Cellularity (MC), Lymphocyte Rich (LR), Lymphocyte Depleted (LD).

HOW TO CITE THIS ARTICLE: Kumar N, Pai RR, Halim I. Reactive milieu of Hodgkin lymphoma with emphasis on mast cells and macrophages. J. Evolution Med. Dent. Sci. 2016;5(64):4504-4507, DOI: 10.14260/jemds/2016/1028

\section{INTRODUCTION}

The diagnosis of Hodgkin Lymphoma (HL) is based on the identification of the diagnostic Reed-Sternberg (RS) cell within an appropriate inflammatory milieu. The neoplastic cell and its variants are present in variable number. The reactive milieu can form up to $99 \%$ of the population in the involved node and is quite heterogeneous consisting of small round lymphocytes, histiocytes, epithelioid histiocytes, plasma cells, neutrophils, eosinophils, and fibroblasts. Based on morphology, phenotype, genotype, and clinical presentation, the latest WHO Classification (2008) is accepted worldwide.(1)

Financial or Other, Competing Interest: None.

Submission 02-07-2016, Peer Review 27-07-2016,

Acceptance 02-08-2016, Published 09-08-2016.

Corresponding Author:

Dr. Nidhish Kumar,

Flat No-302,

Aimaan Residency, B. V. Road,

Opposite Kidzee School, Attavar,

Mangalore-575001, Karnataka.

E-mail: nidhishsingh7@gmail.com

DOI: $10.14260 /$ jemds/2016/1028
The research in HL currently is focused in identifying those cases that are likely to relapse. The advances in treatment are directed towards the background immune cells that give prosurvival feedback to the RS cells. The macrophages and mast cells are intensely investigated to identify their independent prognostic significance and possible target for therapy. This study is an attempt to recognise the importance of mast cells and macrophages in HL.

\section{AIM}

To study the clinical importance of reactive microenvironment in Hodgkin lymphoma with special reference to mast cells and macrophages.

\section{OBJECTIVES}

1. To correlate the reactive background cells, mast cells, and macrophages with B symptoms and stage of the disease.

2. To study the role of mast cell and macrophage counts in the planning of treatment and predicting the treatment outcome. 


\section{METHODOLOGY}

This was a retrospective and prospective study from January 2011 to June 2015 of cases of HL of Department of Pathology, Kasturba Medical College, Mangalore. The Haematoxylin and Eosin (H and $\mathrm{E}$ ) stained slides were reviewed and classified using WHO (2008) classification into 3 main subtypes:1) Nodular Lymphocytic Predominant (NLP)-HL 2) cHL 3) HL-unclassified.(1)

Classical HL was divided into: 1) Nodular Sclerosis (NS)cHL 2) Mixed Cellularity (MC)-cHL 3) Lymphocyte Rich (LR)cHL 4) Lymphocyte Depleted (LD)-cHL.

An overall age and gender distribution was studied. In each of the 4 subgroups also median age and male to female ratio was recorded. Histopathologically in the background, the mast cell count, macrophage count, presence of eosinophilia, neutrophilia, and areas of necrosis were compared within each subgroup. Diagnosis was confirmed by immunohistochemistry. Six immunohistochemical markers were used in the study. CD 15, CD 30, and CD 45 (LCA) were for HL confirmation. CD 3 and CD 20 were for background lymphocytes and CD 68 was for the macrophage count. Giemsa stain was done to highlight the mast cells.

\section{Mast Cell Count(2)}

Mast cells are detected in most of the cases examined and in every histopathological subgroup. A cutoff point of 10 mast cells/10 HPF was chosen. On staining with Giemsa stain, the metachromatic granules of the mast cell stain purple while the background cells stain blue.

\section{Macrophage Count Using CD 68(3)}

The level of CD 68 was recorded and graded with respect to the relative percentage of tumour-associated macrophages in RS cell rich areas. The percentage of macrophages was recorded and graded as 1 if less than $5 \%, 2$ if $5-25 \%$ and 3 if $25-50 \%$ and 4 if more than $50 \%$ of the total cells present in RS cell rich nodules.

\section{RESULTS AND ANALYSIS}

Thirty cases of HL were studied out of which $50 \%$ (15 cases) were NS-cHL, 16.7\% ( 5 cases) were MC-cHL and LD-cHL each, $10 \%$ (3 cases) were LR-cHL, and $6.7 \%$ cases could not be categorised as they were diagnosed on core biopsy.

Out of 30 cases, $43.3 \%$ were in the age group of $21-40$ years, $26.7 \%$ cases were grouped in $41-60$ years, $23.3 \%$ cases were less than 20 years of age, and $6.7 \%$ cases were more than 60 years old respectively.

Nineteen cases were males and 11 were females. Eight cases were in stage I, 11 cases were in stage II, 10 cases were in stage III, and 1 case was in stage IV.

Thirteen (43.3\%) of the cases were CD15 negative and 17 (56.7\%) were CD15 positive. CD30 positivity was seen in 100 $\%$ of cases and CD45(LCA) negativity was noted in $100 \%$ of cases. CD 20 was positive in $7(23.3 \%)$ of cases $(>10 \%)$ and the background lymphocytes were mostly CD 3 positive.

Eleven (73.3\%) out of 15 cases of NS-cHL were in the age group of 21-40 years. Three cases were in age group 20 and below and 1 case was in 41-60 years age group. Cases of LRcHL were seen equally distributed from age 0-60 years while LD-cHL was seen in older age group. Out of 5 cases of LD-cHL, 4 were from $41-60$ years and 1 was above 60 years of age.
Statistical analysis was done and $\mathrm{p}$ value was highly significant. $(\mathrm{p}<0.000)$.

B-symptoms were present in 5 (33.3\%) cases of NS-cHL. Bsymptoms were absent in all cases of MC-cHL. LD-cHL had 5 cases out of which $4(80 \%)$ showed B-symptoms. P value was significant. $(\mathrm{p}<0.033)$.

Six cases showed increased mast cell count. Out of these, 4 (66.7\%) were of NS-cHL and 2 (33.3\%) cases were of LD-cHL. Other cases showed the presence mast cells, but did not show increase in mast cell count.

All cases of LD-cHL showed high macrophage count. Four (80\%) cases showed macrophage count between $25-50 \%$ and $1(20 \%)$ case showed macrophage count $>50 \%$. $\mathrm{P}$ value was significant $(\mathrm{p}<0.046)$.

Nineteen $(63.3 \%)$ cases showed complete effacement of lymph node architecture. Out of these 10 (52.6\%) were of NScHL, $2(10.5 \%)$ were of MC-cHL, 2 (10.5\%) were of LR-cHL, and 5 cases of LD-cHL, which showed complete effacement of lymph node architecture. $P$ value was found to be significant. $(\mathrm{p}<0.011)$.

Eleven (36.7\%) were females and $19(63.3 \%)$ were males. Out of 11 females, 5 (45.5\%) cases were in stage 1 and 6 $(54.5 \%)$ cases were in stage 2 . Out of 19 males, $3(15.8 \%)$ cases were in stage 1, $5(26.3 \%)$ cases were in stage 2, 10 (52.6\%) cases were in stage 3 , and $1(5.3 \%)$ case was in stage 4 . P value was found to be highly significant. $(\mathrm{p}<0.008)$.

Twelve $(40 \%)$ cases showed B-symptoms. Two (16.7\%) cases were in stage 1, $2(16.7 \%)$ cases were in stage 2,7 $(58.3 \%)$ cases were in stage 3 and 1 (18.3\%) case was in stage 4. $P$ value was significant. $(\mathrm{p}<0.039)$.

LDH level was raised in 7 (23.3\%) cases. Four $(57.4 \%)$ were in stage 1 and $1(14.3 \%)$ each in stage 2,3 , and 4 . P value was significant. $(\mathrm{p}<0.037)$.

ESR was raised in $24(80 \%)$ out of 30 cases of HL. Four $(16.7 \%)$ cases were in stage $1,11(45.8 \%)$ cases were in stage $2,8(33.3 \%)$ cases were in stage 3 , and $1(4.2 \%)$ case was in stage 4 . $P$ value was significant. $(\mathrm{p}<0.041)$.

Nineteen $(63.3 \%)$ cases showed complete effacement of lymph node architecture. Two (10.5\%) cases were in stage 1, 8 $(42.1 \%)$ cases were in stage 2, and $9(47.4 \%)$ cases were in stage 3 . P value was highly significant. $(\mathrm{p}<0.006)$

\section{DISCUSSION}

HL is classified as NLPHL and cHL.(1) Classical HL is classified as i) NS-cHL ii) MC-cHL iii) LR-cHL iv) LD-cHL. NLPHL is seen in $4-5 \%$ of cases of HL.(1,3) NLPHL differs greatly in morphology, phenotype, genotype, and clinical behaviour from cHL. (4) The only feature common between NLPHL and cHL is the low number of neoplastic RS cells.(4) As compared to the bimodal peak in cHL, NLPHL has a unimodal peak in $4^{\text {th }}$ decade of life and affects mostly single group of lymph nodes (Axillary, cervical, or inguinal) with occasional bone marrow involvement.(4) In our study, there were no cases of NLPHL and HL-unclassified.

NS-cHL is the most common subtype.(1) It corresponds to $78-80 \%$ of all HL cases in developed countries; however, the incidence of the subtypes vary greatly in other geographical areas.(1,5) NS-cHL subtype is followed by MC-cHL, which comprises of $15-25 \%$ of cases of cHL.(1) MC-cHL is followed by LR-cHL, which is seen in $4 \%$ of cases. LD-cHL is the least commonly found subtype occurring in about $1 \%$ of cases of HL. $(1,4,6)$ 


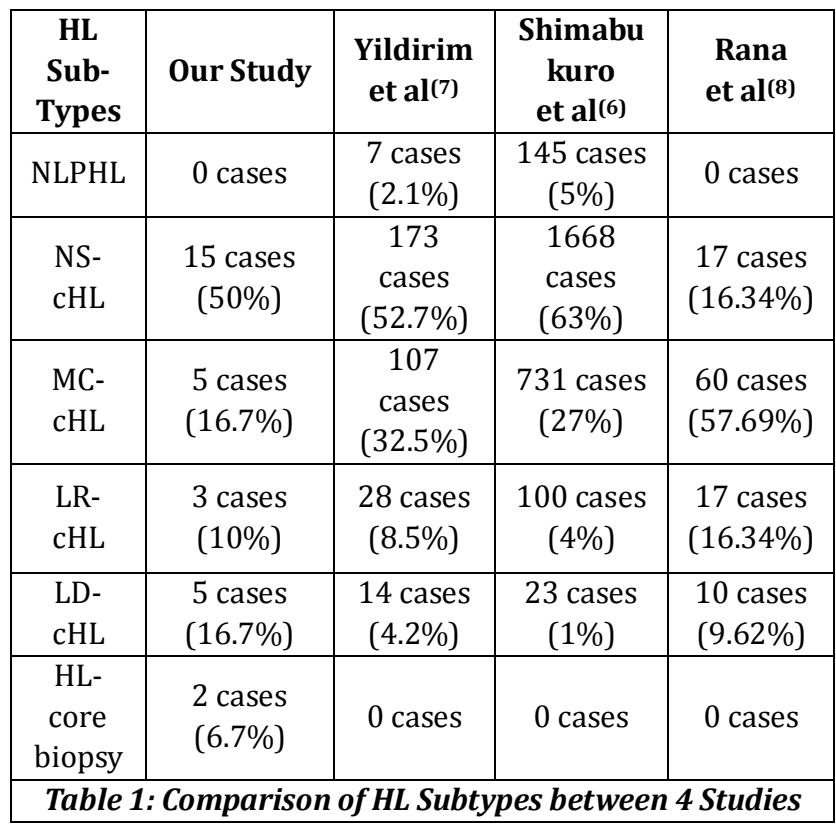

\section{Mast Cells in HL}

Mast cells act actively in various types of cancers. They can either have a pro-tumorigenic function or an anti-tumorigenic function depending on the type of cancer. Mast cells were seen in all cases of cHL. In our study, all cases showed presence of mast cells, but only $6(20 \%)$ cases showed increase in mast cell count of $>10$ mast cells $/ 10$ hpf. Out of 6 cases, 4 (66.7\%) cases were of NS-cHL and 2 (33.3\%) cases were of LD-cHL. In a study done by Molin et al, they described that mast cells were seen in the proximity to RS cells.(2) No such observation was made in our study.

On 5 cases of reactive follicular hyperplasia of lymph node, Giemsa stain was done as a control and mast cell count was noted in all. Mast cell count was found high in all cases. Around 25-30 mast cells/10 HPF were found in each case.

A correlation between the number of mast cells and poor prognosis was demonstrated in a number of studies. In a study done by Molin et al(2) on 123 patients, 71 (58\%) cases had $>10$ mast cells $/ 10$ HPF and had a significant worse disease free survival $(p<0.01) .{ }^{(9)}$ It was also seen that most of the cases, which had increased mast cell count were of NS-cHL.

A quantitative study of mast cells on 45 cases of Hodgkin's disease was done by Crocker and Smith.(10) It was found that NS-cHL cases contained many more mast cells as compared to other subtypes of HL.(11) A similar observation was made in our study. Out of 6 cases, which showed increase in mast cell count, 4 were of NS-cHL. They also concluded that mast cells were more in the fibrous areas than in the cellular zones. In our study, among 4 cases that showed increased mast cell count. In 2 cases, fibrous area showed slightly more number of mast cells than the cellular areas.

The mean number of mast cells in 4 cases of NS-cHL with raised count was 14.25 . When compared to a study done by Molin et al, it was found to be 22.6. In another study done by Crocker and Smith using Astra blue stain mean number of mast cells in NS-cHL per 20 fields (10 fibrous and 10 cellular areas) (10x) was found to be 57. Thus, it was concluded in our study that probably degranulated mast cells are not stained by the Giemsa stain. CD 117.(12) CD 25.(12) tryptase immunostaining.(12) and Astra blue.(11) are the special stains that can be used to study the mast cells. Future studies on mast cells can be done using more specific markers/stains.

\section{Macrophage Count in HL}

Macrophages were counted and grouped under 4 categories, which were $0-5 \%, 5-25 \%, 25-50 \%$, and $>50 \%$ of the total cell population/HPF.

Ten $(66.7 \%)$ cases of NS-cHL showed macrophage count from $5-25 \%$ and only 3 (33.3\%) cases showed count from 25$50 \%$. Out of 5 cases of MC-cHL, $4(80 \%)$ cases were in the range of $5-25 \%$ and only 1 (20\%) case showed count from $25-50 \%$. LR-cHL had a case each in $0-5 \%, 5-25 \%$, and $25-50 \%$. Four $(80 \%)$ cases of LD-cHL showed macrophage count between $25-50 \%$ and 1 (20\%) case showed macrophage count $>50 \%$ correlating with the aggressive nature and advanced stage of the disease.

Although, most studies have demonstrated an association between inferior outcome in cHL and increased tumour associated macrophages, a variability has been seen in the suggested threshold values for CD 68.(13) This variability is likely due to difference in IHC quantitation methodology as most of the studies are done using manual visual scoring and there is lack of subsequent validation of thresholds in the studies.(13)

The potential pitfall in the use of IHC biomarkers in routine practice is lack of reproducibility and inconsistency of visual or manual scoring system.(13)

Poor inter-observer agreement for low scores with CD 68 was shown by Azambuja et al.(3) These issues are overcome by the use of computer image analysis to produce greater objectivity in scoring. Computer-assisted stereological analysis was done by Kamper et al(11) and point grid counting methodology was used for assessing CD 68 count, which showed increased CD 68 expression with inferior outcome in cHL.(11) However, this method was labour intensive.(11)

In the study done by Harris et al,(14) CD68 and CD163 IHC stains were used to study the macrophage count of 44 cases of cHL. It was observed that though CD68 is a marker of cells of monocyte/macrophage lineage. It is relatively nonspecific with reported staining in neoplasms like melanoma, carcinoma, lymphoma, angiosarcoma, and schwannoma. There is also nonspecific staining seen in inflammatory cells and fibroblasts with CD68. CD163, which when compared to CD68 was found to be highly specific for tumours of monocyte/macrophage lineage with less background staining in HRS rich areas. CD163 may be used in future for the macrophage count in HL.

In the same study on 44 cases, it was found that a low CD68 score was associated with $100 \%$ disease-specific survival in patients with limited stage disease (Stage I and IIa).

In a study done by Steidl et al(15) on 130 cases, it was seen that increased number of CD68 positive macrophages were associated with increased likelihood of relapse and shortened progression and disease free survival. It was also seen that in absence of elevated number of CD68 positive macrophages with limited stage disease the long-term disease-specific survival was $100 \%$ with a conclusion that increased number of tumour associated macrophages were associated strongly with shortened survival in patients with cHL.

In another study done by Kamper et al(11) on 208 cases, CD68 and CD163 expression was assessed with the degree of macrophage infiltration in the tumour. 
It was seen at the univariate level, high CD68 and CD163 expression correlated with poorer overall survival and event free survival. Even at multivariate level, high CD68 expression was significant in predicting the overall survival. It was also demonstrated that high levels of CD68 and CD163 were associated with the presence of EBV in the tumour cells. EBV stains were not done in our study.

A study was done by Tan et al(13) on 287 patients using CD68 and CD163 as IHC markers. The CD68 and CD163 expression were analysed by computer image analysis and optimum thresholds for overall survival were determined. It was seen that increased CD68 and CD163 expression was associated with inferior failure free survival and poor prognosis in cHL. Increased CD163 expression was also associated with increased age and MC-cHL.

In a study done by Azambuja et al(3) on 256 cases of cHL, CD68 and CD163 expression with clinical outcomes was studied. The patients were uniformly treated with ABVD chemotherapy. No association between clinical characteristics and CD68 and CD163 expression was seen. However, high level of CD68 and CD163 was associated with the presence of EBV in Hodgkin tumour cells. Thus, it was concluded that CD68 and CD163 expression needs to be further evaluated before they can be used for prognostic stratification.

In our study, after the follow up of 12 months, it was found that all patients responded well to ABVD chemotherapy and relapse was seen in only 1 (3.3\%) case of NS-cHL in which the macrophage count was in the lower range i.e. between 5-25\%. The main pit fall of our study was that only a follow up of 12 months was available in most of the cases thus no conclusive comment on the treatment outcome, prognosis, and relapse can be made. A longer followup is needed to correlate these parameters.

\section{CONCLUSION}

In this study of microenvironment of HL mast cells and macrophages were analysed in each subtype. Though the mast cells were seen in all cases, an increased count of $>10 / 10$ HPF was observed only in 6 cases with the highest count being 22 mast cells/10 hpf in LD-cHL. The mast cell numbers did not correlate with B-symptoms and stage of the disease.

CD68 was used to highlight the macrophages. The macrophage count was highest in LD-cHL and was statistically significant and thus correlated with this aggressive subtype of HL. The macrophage count did not correlate with B-symptoms and stage of the disease.

All patients were treated with ABVD chemotherapy. In a follow up of 12 months in 27 patients, 26 were free of clinical disease. Only one patient had relapse in which the mast cell and macrophage count was in lower range. A conclusion on survival versus the macrophage count and mast cell count was not possible in this study because of shorter follow up. A longer follow up and more number of cases have to be studied to validate the role of mast cells and macrophages in the prognosis of HL. Mast cell and macrophages may have to be done routinely in future to plan the treatment and predict the treatment outcome.

\section{REFERENCES}

1. Piccaluga PP, Agostinelli C, Gazzola A, et al. Pathobiology of Hodgkin lymphoma. Adv Haematol 2011;2011:920898.

2. Molin D, Edstrom A, Glimelius I, et al. Mast cell infiltration correlates with poor prognosis in Hodgkin's lymphoma. British Journal of Haematology 2002;119(1):122-4.

3. Azambuja D, Natkunam Y, Biasoli I, et al. Lack of association of tumour-associated macrophages with clinical outcome in patients with classical Hodgkin's lymphoma. Ann Oncol 2012;23(3):736-42.

4. Pileri SA, Ascani S, Leoncini L, et al. Hodgkin's lymphoma: the pathologist's viewpoint. J Clin Pathol 2002;55(3):16276.

5. Kumar V, Abbas A, Aster J. Robbins and Cotran pathologic basis of disease: South Asia edition. 9th ed. New Delhi: Elsevier 2014.

6. Shimabukuro-Vornhagen A, Haverkamp H, Engert A, et al. Lymphocyte-rich classical Hodgkin's lymphoma: clinical presentation and treatment outcome in 100 patients treated within German Hodgkin's study group trials. J Clin Oncol 2005;23(24):5739-45.

7. Yıldırım M, Yıldız M, Kurtoğlu E, et al. Epidemiology and histological subtypes of Hodgkin lymphoma in the SouthWest of Turkey. Gulhane Med J 2012;54(3):216-9.

8. Rana R, Chopra R, Masih K, et al. Hodgkin's disease: a clinicopathologic study. Indian J Pathol Microbiol 1995;38(3):245-9.

9. Glimelius I. Hodgkin lymphoma-an interplay between tumour cell and microenvironment. Review based on doctoral thesis. Uppsala University 2009;449:1-66.

10. Crocker J, Smith PJ. A quantitative study of mast cells in Hodgkin's disease. J Clin Pathol 1984;37(5):519-22.

11. Kamper P, Bendix K, Hamilton-Dutoit S, et al. Tumourinfiltrating macrophages correlate with adverse prognosis and Epstein-Barr virus status in classical Hodgkin's lymphoma. Haematologica 2011;96(2):269-76.

12. Gasljevic G, Grcar-Kuzmanov B, Grosel A, et al. Hodgkin's lymphoma is a rare form of clonal haematological nonmast cell disease in systemic mastocytosis. Diagn Pathol 2015;10:5.

13. Tan KL, Scott DW, Hong F, et al. Tumour-associated macrophages predict inferior outcomes in classic Hodgkin lymphoma: a correlative study from the e2496 intergroup trial. Blood 2012;120(16):3280-7.

14. Harris JA, Jain S, Ren Q et al. CD163 versus CD68 in tumour-associated macrophages of classical Hodgkin lymphoma. Diagn Pathol 2012;7:12.

15. Steidl C, Lee T, Shah SP, et al. Tumour-associated macrophages and survival in classic Hodgkin's lymphoma. N Engl J Med 2010;362(10):875-85. 